\title{
Erratum to: Common Borel Radii of an Algebroid Function and Its Derivative
}

Nan Wu and Zu-xing Xuan

\section{Erratum to: Results Math}

\section{DOI 10.1007/s00025-011-0132-y}

The affiliations were incorrect. The correct information is given below.

Nan $\mathrm{Wu}$ and Zu-xing Xuan*

Department of Mathematical Sciences

Tsinghua University

Beijing 100084

People's Republic of China

e-mail: wunan07@gmail.com

Zu-xing Xuan*

Basic Department

Beijing Union University

Beijing 100101

People's Republic of China

e-mail: xuanzuxing@smss . buaa.cn;

xuanzuxing@ss. buaa.edu.cn

\footnotetext{
* Corresponding author.
}

The online version of the original article can be found under doi:10.1007/s00025-011-0132-y. 